\title{
Experimental and Simulation Methods in Scanning Electron Nanobeam Diffraction
}

Colin Ophus ${ }^{1}$, Karen Bustillo ${ }^{1}$, Thomas C Pekin ${ }^{1}$, AJ Pryor $\mathrm{Jr}^{2}{ }^{2}$, Jianwei Miao ${ }^{2}$, Brian Shevitski ${ }^{1}$, Shaul Aloni $^{1}$, Peter Ercius ${ }^{1}$, Jim Ciston ${ }^{1}$, and Andrew M Minor ${ }^{1}$

1. Molecular Foundry, Lawrence Berkeley National Laboratory, Berkeley USA.

2. Department of Physics and Astronomy, University of California Los Angeles, Los Angeles USA.

Traditional scanning transmission electron microscopy (STEM) detectors are large, single pixels that integrate a subset of the transmitted electron beam signal scattered from each electron probe position. These transmitted signals are extremely rich in information, containing localized information on sample structure, composition, phonon spectra, three-dimensional defect crystallography and more. Conventional STEM imaging experiments record only 1-2 values per probe position, throwing away most of the diffracted signal information. With the introduction of extremely high speed direct electron detectors, we can now record a full image (2D data) of the diffracted electron probe scanned over the sample (2D grid of positions), producing a four-dimensional dataset we refer to as a 4D-STEM experiment. In this tutorial, we will describe the challenges and opportunities created by 4D-STEM, and explain in detail the nuts and bolts of some 4D-STEM experiments and simulation methods.

Figure 1a shows the basic geometry of a 4D-STEM experiment. A converged electron probe with a size ranging from sub-Ångstrom to multiple nanometers is focused upon the sample surface. The probe scatters from the sample volume, forming a convergent beam electron diffraction (CBED) pattern. This pattern is recorded with a high speed direct electron detector (a pixelated camera), and then the probe is moved to the next position on the sample surface. Electrons scattered to higher angles are often also recorded using an annular detector, generating another signal channel typically referred to as a high angle annular dark field (HAADF) image.

Figures $1 \mathbf{b}$ and $\mathbf{c}$ show typical 4D-STEM experiments. The sample imaged in Figure $1 \mathbf{b}$ is a semicrystalline soft-matter sample, where overlapping crystalline domains produce diffraction spots perpendicular to the backbone of the structure. Automated peak-finding codes are used to find and classify these diffracted disks. From a large dataset of classified diffraction peaks, the underlying grain structure can be visualized, as above. Figure 1c shows strain mapping from a stainless-steel sample, adapted from [1]. In this experiment, each probe position produces a grid of disks that correspond to the reciprocal lattice vectors. By measuring the reciprocal lattice with high precision, we can measure small lattice distortions on the scale of individual STEM probe positions, ranging from nanometers to micrometers. In this talk, I will also show 4D-STEM strain measurements from amorphous samples.

Figure 1d shows the algorithmic steps required for a STEM simulation, using either the multislice method or the newly developed PRISM algorithm [2,3]. First, we build a model of the atomic positions for a given sample, and then use tabulated atomic scattering factors to generate projected potentials. Next, we define the initial electron probes and propagate them through the sample. Finally, we can save these outputs as 2D, 3D or 4D output files. In this talk I will demonstrate both simulation methods using the Prismatic code [3]. Figure 1e shows a study where experimental and simulated CBED patterns were matched to determine the best fit for sample thickness and composition in an STO-LMO multi-layer sample [4]. This was done by simulating a large library of possible CBED patterns. Using this example and others, I will show how simulation can help with the interpretation of 4D-STEM experiments [5]. 


\section{References}

[1] TC Pekin, C Gammer, J Ciston, AM Minor and C Ophus 176, 170 (2017).

[2] C Ophus, Advanced Structural and Chemical Imaging 2, 11 (2017).

[3] AJ Pryor Jr., C Ophus and Jianwei Miao, Advanced Structural Imaging 3, 15 (2017).

[4] C Ophus, P Ercius, M Huijben and J Ciston, Applied Physics Letters 110, 063102 (2017).

[5] Work at the Molecular Foundry was supported by the Office of Science, Office of Basic Energy Sciences, of the U.S. Department of Energy under Contract No. DE-AC02-05CH11231. We thank the NVIDIA Corporation for donation of GPU resources.

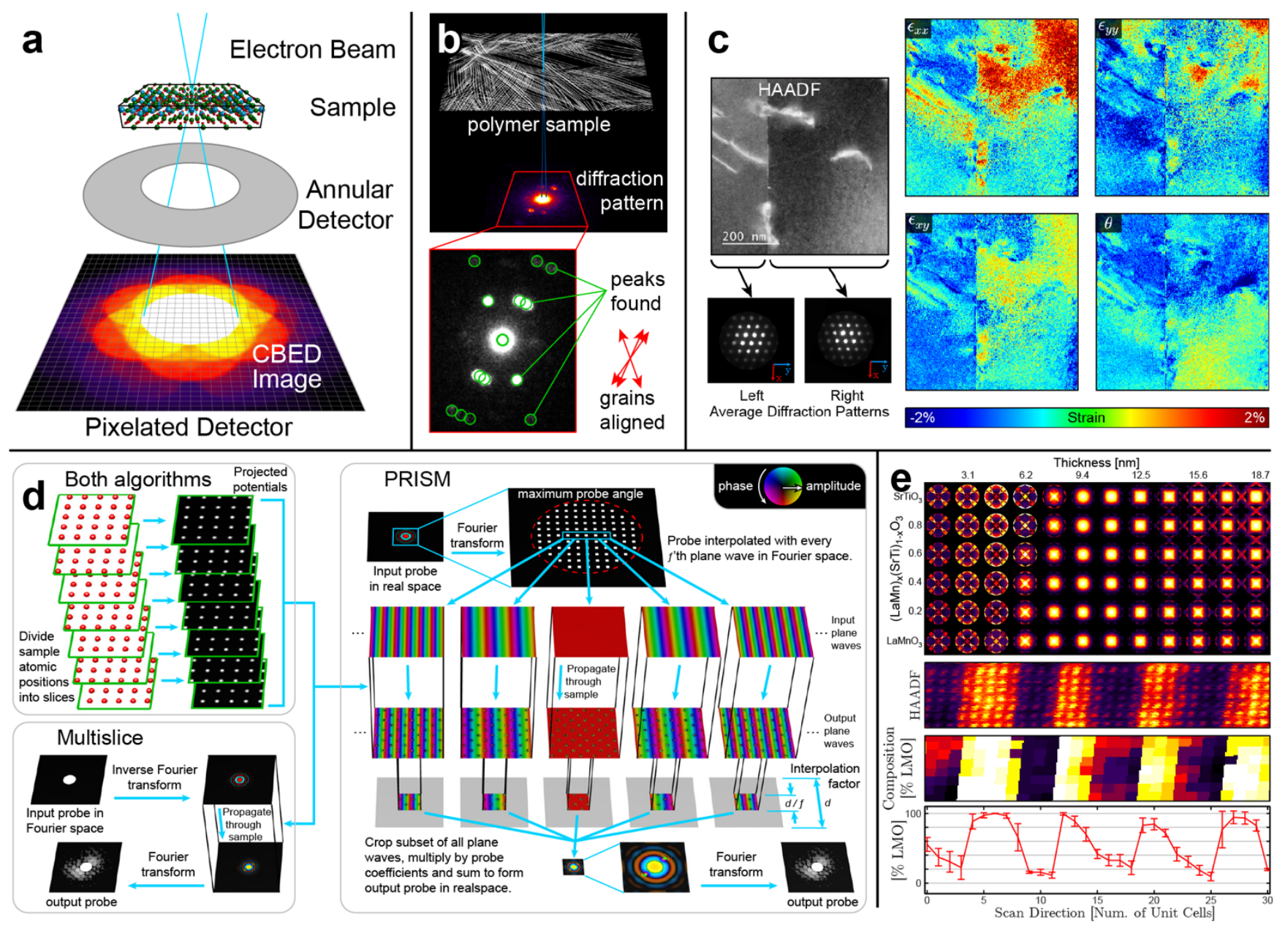

Figure 1. (a) Experimental geometry of a 4D-STEM experiment measuring a convergent beam electron diffraction (CBED) pattern, also known as pixelated STEM or scanning electron nanodiffraction (SEND). (b) Experimental diffraction pattern from a weakly scattering polymer material, where diffraction peaks are automatically detected and classified to measure orientation. (c) Strain of local lattice displacements across a twin boundary in a crystal, measured from slight shifts in the diffracted disk positions, adapted from [1]. (d) Simulation steps for two algorithms for computing 4D-STEM patterns: multislice and PRISM, adapted from [2, 3]. (e) Diffraction pattern library computed for different sample compositions and thicknesses, used the classify composition of each unit cell in the experimental 4D-STEM dataset below, adapted from [4]. 